\title{
Synergy between intraepithelial lymphocytes and lamina propria T cells drives intestinal inflammation during infection
}

\author{
CE Egan ${ }^{1}, \mathrm{KJ} \mathrm{Maurer}^{2,3}$, SB Cohen $^{1}, \mathrm{M} \mathrm{Mack}^{4}, \mathrm{KW}$ Simpson $^{5}$ and EY Denkers ${ }^{1}$
}

Oral infection of C57BL/6 mice with Toxoplasma gondii triggers severe necrosis in the ileum within 7-10 days of infection. Lesion development is mediated by Th- 1 cytokines, CD4 ${ }^{+} \mathrm{T}$ cells, and subepithelial bacterial translocation. As such, these features share similarity to Crohn's disease. Recently, we uncovered a role for intraepithelial lymphocytes (IELs) in mediating pathology after Toxoplasma infection. We show here that $\alpha \boldsymbol{\beta}$ and not $\gamma \boldsymbol{\delta}$ T-cell IELs mediate intestinal damage. By adoptive transfer of mucosal T cells into naive Rag ${ }^{-/-}$mice, we demonstrate that IELs do not function alone to cause inflammatory lesions, but act with $\mathrm{CD} 4^{+} \mathrm{T}$ lymphocytes from the lamina propria (LP). Furthermore, recipient mice pretreated with broad-spectrum antibiotics to eliminate intestinal flora resisted intestinal disease after transfer of IELs and LP lymphocytes. Our data provide valuable new insights into the mechanisms of intestinal inflammation, findings that have important implications for understanding human inflammatory bowel disease.

\section{INTRODUCTION}

Inflammatory bowel diseases (IBDs) in humans are chronic inflammatory disorders that most often emerge during the second or third decade of life. Crohn's disease and ulcerative colitis are the most common types of idiopathic IBDs. ${ }^{1}$ Crohn's disease affects the terminal ileum in more than two-thirds of patients, and is characterized by transmural inflammation and macrophage aggregation forming noncaseous granulomas. The incidence of IBD, particularly Crohn's disease, has been increasing in recent decades in developed regions of the world. ${ }^{2}$ Although the etiology of IBD is unclear, a combination of genetic and environmental factors is believed to lead to dysregulated immunity, altered intestinal barrier function, and dysfunctional responses to the gut flora. ${ }^{3}$

IBDs in mice can be induced genetically, chemically, and by certain infections. Included in the latter group is infection with the protozoan parasite Toxoplasma gondii, a microorganism infecting up to $30-50 \%$ of the human population. ${ }^{4}$ Although normally asymptomatic, Toxoplasma emerges as an opportunistic pathogen in immunodeficient populations. ${ }^{5}$ The parasite induces a strong Th1 response. ${ }^{6}$ Oral infection of certain inbred mice, typified by the C57BL/6 strain, triggers proinflammatory pathology centered in the ileum that is characterized by necrosis of the villi and mucosal cells. ${ }^{7,8}$ Lesion development is dependent on $\mathrm{CD}^{+}{ }^{+} \mathrm{T}$ cells, interferon (IFN)- $\gamma$, and tumor necrosis factor (TNF)- $\alpha$, and is therefore primarily a Th-1-mediated disease. Endogenous intestinal bacteria are involved in lesion induction, because infection is associated with a shift in the gut flora to predominantly Gram-negative Escherichia coli, increased adherence, and subepithelial bacterial translocation. Moreover, gut flora-depleted, as well as Tlr $4^{-/-}$and $T l r 9^{-/-}$mice, are protected from parasite-induced ileitis. ${ }^{9-11}$ Overall, as an IBD model, T. gondii-induced ileitis bares many striking similarities to Crohn's disease. ${ }^{12}$

We recently uncovered a role for chemokine (C-C motif) receptor (CCR) 2-dependent intraepithelial lymphocytes (IELs) in parasite-induced ileitis during T. gondii infection. ${ }^{13}$ By transferring wild-type (WT) IELs into C $\mathrm{Cr} 2^{-/-}$mice, we converted the knockout strain from a resistant to susceptible phenotype with regard to induction of small intestinal lesions. These results were of great interest because, although some studies suggest that IELs can have a pathogenic role in intestinal inflammatory

\footnotetext{
${ }^{1}$ Department of Microbiology and Immunology, College of Veterinary Medicine, Cornell University, Ithaca, New York, USA. ${ }^{2}$ Department of Biomedical Sciences, College of Veterinary Medicine, Cornell University, Ithaca, New York, USA. ${ }^{3}$ Center for Animal Resources and Education, College of Veterinary Medicine, Cornell University, Ithaca, New York, USA. ${ }^{4}$ Department of Internal Medicine II, University of Regensburg, Regensburg, Germany. ${ }^{5}$ Department of Clinical Sciences, College of Veterinary Medicine, Cornell University, Ithaca, New York, USA. Correspondence: CE Eganor or EY Denkers (cee22@cornell.edu or eyd1@cornell.edu) 
disorders, ${ }^{14,15}$ these lymphocytes are more commonly associated with homeostasis in the gut and maintenance of barrier function. ${ }^{16}$

Here, we show that among IELs, those expressing $\alpha \beta$ T-cell receptor (TCR) mediate disease, whereas $\gamma \delta$ TCR IELs are inert in this regard. We develop and validate a new model of parasite-induced ileitis, in which transfer of IELs in combination with lamina propria (LP) cells triggers rapid ileitis in immunodeficient $R a g 1^{-/-}$recipients without the presence of parasites. We show that intestinal microflora in recipient mice is essential for development of ileitis after adoptive transfer in this model. Furthermore, we show that IELs recruit LP CD4 ${ }^{+}$T lymphocytes into intraepithelial regions of the mucosa, and that these cells are effectors of intestinal damage. Our data shed new light on the pathogenesis of small intestinal inflammation triggered by microbial infection, and they have important implications with regard to understanding human IBDs.

\section{RESULTS}

Peroral Toxoplasma infection triggers rapid conversion to a proinflammatory cytokine profile in the small intestine

T. gondii infection in C57BL/6 strain mice induces proinflammatory pathology in the small intestine ileum. ${ }^{8}$ Here, we measured cytokines in overnight cultures of ileal biopsy samples collected over a time course of infection (Figure 1). Increasing amounts of IFN- $\gamma(\mathbf{a})$, TNF- $\alpha$ (b), and IL-12/23p40 (c) were detected as infection progressed in biopsy samples with and without Peyer's patches. Examination of transcriptional responses in the ileum further confirmed these data, because transcripts for If $n-\gamma(\mathbf{d})$, $\operatorname{Tnf}-\alpha(\mathbf{e})$, and $I l-12 p 40$ (f) increased in the ileal tissue over the
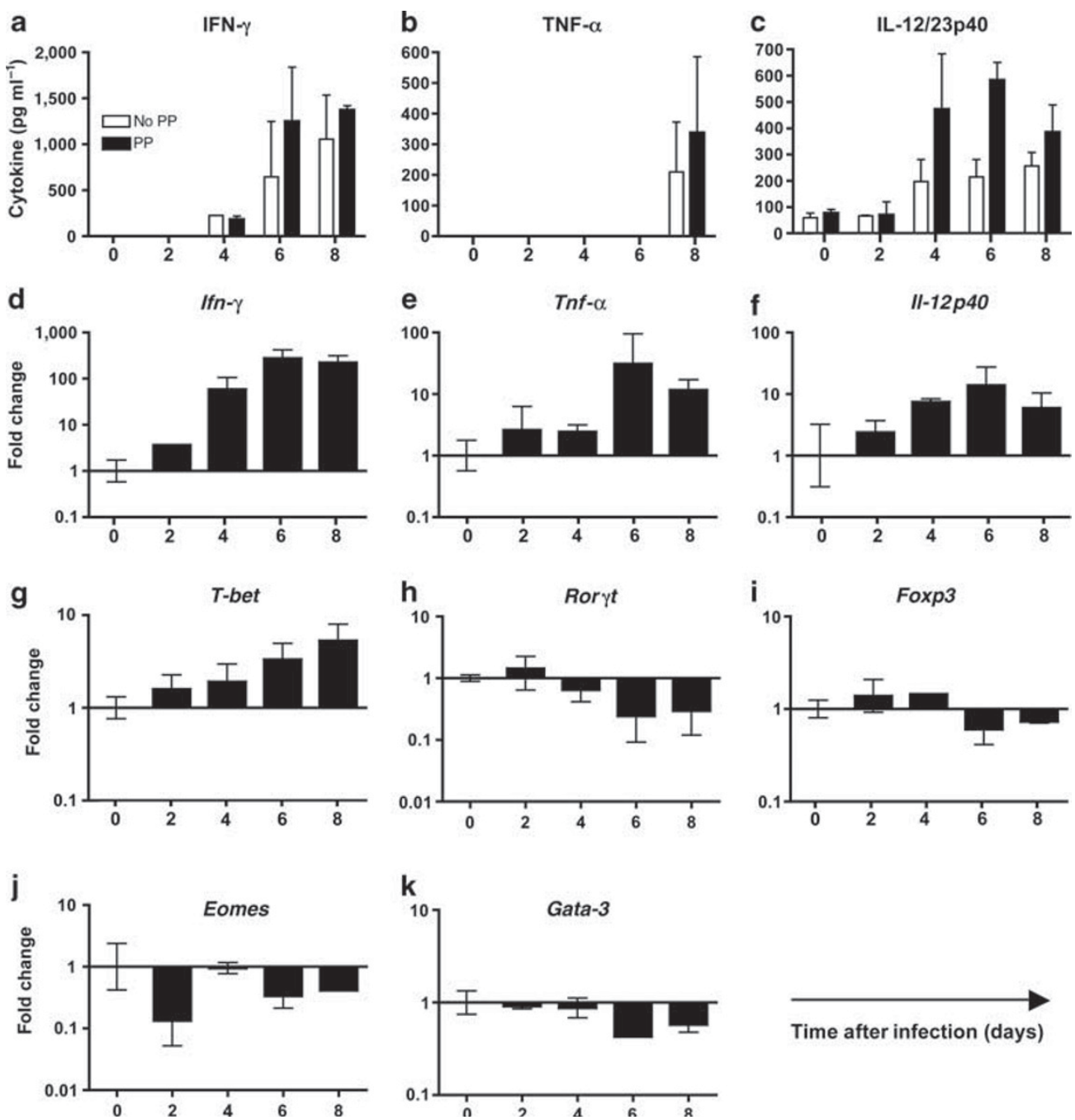

Figure 1 Emergence of a proinflammatory cytokine profile in the small intestine during T. gondii infection. Biopsy samples of the small intestine with or without Peyer's patches (PP) were cultured overnight and supernatants assayed for (a) IFN- $\gamma$, (b) TNF- $\alpha$, and (c) IL-12/23p40 by ELISA. Data, representative of two independent experiments, are plotted as the mean and s.e. between individual mice $(n=3)$. Real-time RT-PCR analysis of intestinal biopsy samples revealed increased transcription genes encoding (d) Ifn- $\gamma$, (e) Tnf- $\alpha$, and (f) II-12p40. Examination of the T-cell transcription factor T-bet revealed a steady increase over the course of infection ( $\mathbf{g})$ and a concomitant decrease in Roryt expression (h). Transcription factors (i) Foxp3, (j) Eomes, and (k) Gata-3 showed no consistent change or decreased expression over the course of infection. These experiments were repeated twice with similar results. ELISA, enzyme-linked immunosorbent assay; IFN- $\gamma$, interferon- $\gamma$; IL, interleukin; RT-PCR, reverse transcriptase-PCR; TNF- $\alpha$, tumor necrosis factor- $\alpha$. 
course of infection with similar kinetics to that seen with cytokine secretion. These data are consistent with previous studies that have shown involvement of each of these cytokines in Toxoplasmainduced immunopathology in the small intestine.,

We also examined expression of T-cell master transcriptional factors. The Th-1 transcription factor, $T$-bet, increased steadily over the course of infection (Figure 1g). Interestingly, this was paralleled by a decrease in Roryt, a master regulator of Th- 17 cells (Figure 1h). Transcription factors associated with Treg cells (Foxp3; Figure 1i), $\mathrm{CD}^{+}$cytotoxic T cells (Eomes; Figure 1j), and Th-2 (Gata-3; Figure 1k) remained relatively unchanged or showed no consistent pattern over the time course of infection. Overall, the data show that Toxoplasma induces a dominant Th-1-type response in the ileum.

\section{TCR $\alpha \beta^{+}$but not TCR $\gamma \delta^{+}$IELs mediate inflammation in infected mice}

Previously, we along with others showed that $\mathrm{Ccr}^{-/-}$mice are protected from T. gondii-induced ileitis ${ }^{13,17}$ (Supplementary Figure S1 online). Importantly, transfer of parasite-elicited WT IELs to infected $\mathrm{C} c 2^{-/-}$mice triggered ileal damage. ${ }^{13}$ The majority of IELs express CD8, but the cells can be subdivided into TCR $\alpha \beta$ - and TCR $\gamma \delta$-positive sub-populations (Figure 2a). To examine whether the pathological activity of parasite-induced IELs could be attributed exclusively to either of these populations, we separated IELs from day 4 -infected WT mice into TCR $\alpha \beta$ (Figure 2b) and TCR $\gamma \delta$ (Figure 2c) populations, adoptively transferred (AT) these cells into day 4-infected $\mathrm{Ccr}^{-/-}$animals, and then collected tissues 5 days later for histopathological evaluation. As shown in Figure $2 \mathbf{d}$ and $\mathbf{f}$, mice receiving TCR $\alpha \beta^{+}$IELs developed ileitis resembling that occurring in WT mice, marked by fusion, necrosis, sloughing of villus tips, and subepithelial inflammation. In marked contrast, mice receiving TCR $\gamma \delta^{+}$IELs failed to display severe lesion development (Figure $2 \mathbf{e}$ and $\mathbf{f}$ ), although some mild inflammatory changes were found in these animals (villus fusion being the most notable and visible in Figure 2e). This result is consistent with others showing a lack of involvement of TCR $\gamma \delta^{+}$T lymphocytes in Toxoplasma-triggered ileitis. ${ }^{8}$

\section{Emergence of a CCR2 + CD4 + IEL population is coincident with maximal inflammation}

Our data associate Toxoplasma-induced ileitis with IELs, the majority of which are CD8 positive. Yet, studies in experimental models of colitis, and in Crohn's disease, implicate a prominent role for $\mathrm{CD} 4^{+} \mathrm{T}$ lymphocytes in damage to the intestine. ${ }^{3,18}$ To investigate this issue, we examined changes in T-cell populations in the IEL compartment over the course of infection in WT mice. IELs were gated on the expression of CCR2 (Figure 3a), and then the expression of CD8 $\alpha$ and CD4 was examined in CCR2-positive and CCR2-negative populations. IELs isolated from naive and day 4 post-infection mice displayed a similar expression pattern for CD8 $\alpha$ and $\mathrm{CD} 4$ and other T-cell markers among CCR2-positive and CCR2-negative cells. However, by day 8 post infection, there was a dramatic increase in $\mathrm{CD} 4{ }^{+}$IELs, with the $\mathrm{CD} 4{ }^{+} \mathrm{CCR} 2{ }^{-}$population
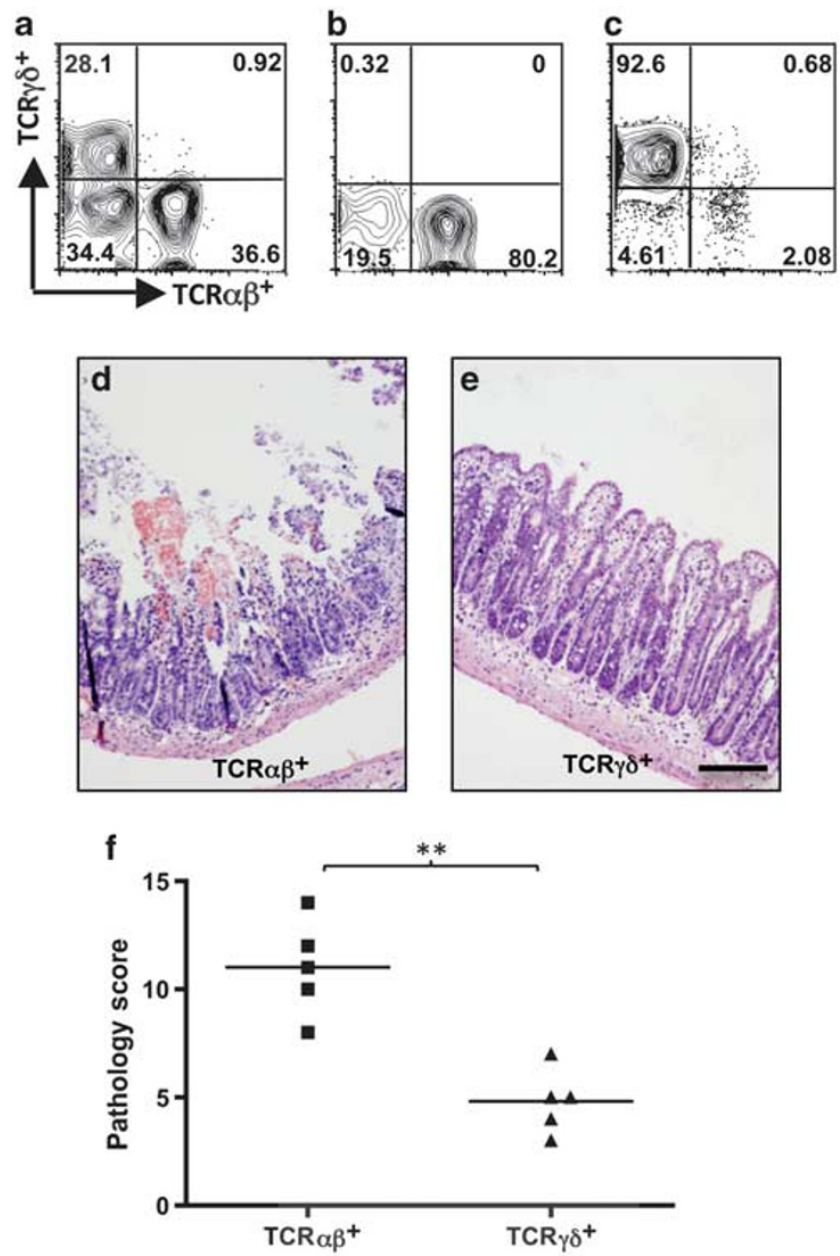

Figure $2 \mathrm{TCR} \alpha \beta^{+}$and not TCR $\gamma \delta^{+}$IELs from WT mice induce damage in $\mathrm{Ccr}^{-/-}$hosts. IELs were collected from WT day 4-infected mice (a) and fractionated into TCR $\alpha \beta^{+}$(b) and TCR $\gamma \delta^{+}$(c) sub-populations by antibody labeling and magnetic bead separation. Day 4-infected CCr2 ${ }^{-/-}$mice received either TCR $\alpha \beta^{+}$IELs or TCR $\gamma \delta^{+}$IELs, and 5 days later intestines were collected and examined for pathological changes. (d, e) Images of $\mathrm{CCr} 2^{-/-}$mice receiving TCR $\alpha \beta^{+}$and TCR $\gamma \delta^{+}$IELs, respectively. Scale bar $=50 \mu \mathrm{m}$; (e). (f) Represents pathology scores for individual mice ( $n=5$ per group), ${ }^{\star \star} P<0.05$. The experiment was repeated twice with the same result. IEL, intraepithelial lymphocyte; TCR, T-cell receptor; WT, wild type.

increasing 3-fold and CD4 ${ }^{+}$CCR2 ${ }^{+}$cells increasing 10 -fold (Figure 3b).

Emergence of CCR2 ${ }^{+} \mathrm{CD} 4{ }^{+}$cells in the IEL compartment suggested that recruitment was CCR2 dependent. Accordingly, we examined recruitment of $\mathrm{CD} 4^{+}$cells in $\mathrm{Ccr}^{-/-}$mice at day 8 post infection. In comparison with IELs from infected WT mice, there was a substantial defect in CD4 cell recruitment in the absence of CCR2 (Figure 3c). Over multiple experiments comparing WT and $\mathrm{Ccr} 2^{-/-}$mice, there was $~ 50 \%$ decreased recruitment of $\mathrm{CD} 4$ cells associated with the lack of CCR2 (Figure 3c). Virtually all CD $4^{+}$IELs expressed TCR $\alpha \beta$ (Figure 3d). In contrast, CD $8 \alpha^{+}$IELs expressed either $\alpha \beta T C R$ or $\gamma \delta$ TCR (Figure 3d). 
b

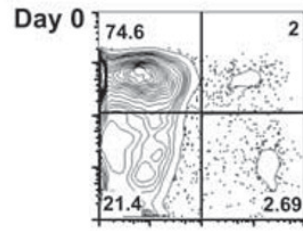

a

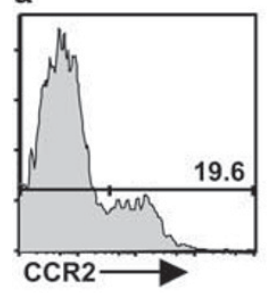

Day 4
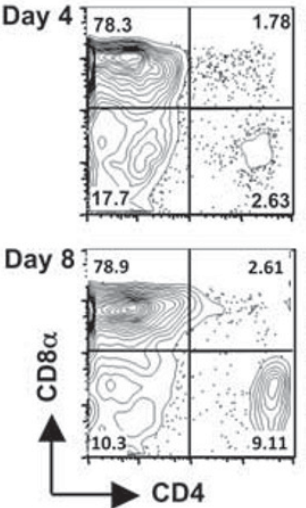

$\mathrm{CCR}^{+}$
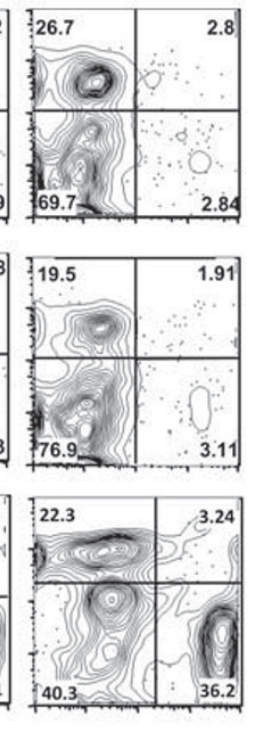

c
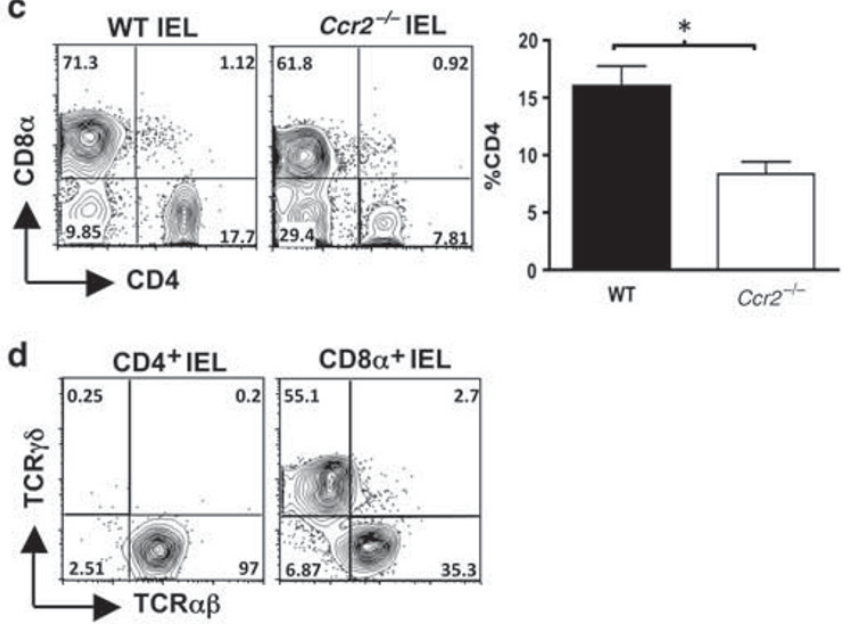

Figure 3 Emergence of CCR2 ${ }^{+} \mathrm{TCR} \alpha \beta^{+} \mathrm{CD} 4^{+}$IELs correlates with maximum pathology during Toxoplasma infection. IELs were isolated and stained with anti-CCR2 mAb (a, CCR2 expression on IELs from day 4-infected mice), in combination with Ab-specific for CD4, CD8 $\alpha, C D 8 \beta, \alpha \beta T C R$, and $\gamma \delta$ TCR. (b) Respective expression of CD $8 \alpha$ and CD4 in CCR2 ${ }^{-}$and CCR2 ${ }^{+}$populations over the course of infection. Essentially identical results were obtained in three independent experiments. To examine whether Toxoplasma-triggered recruitment of TCR $\alpha \beta^{+} \mathrm{CD} 4^{+}$to the IEL compartment is dependent on CCR2, IELs were isolated from WT and Ccr2 ${ }^{-/-}$mice 8 days after infection and expression of CD8 $\alpha$ and CD4 was assessed. Representative staining profiles are shown in (c), which also shows the average percentage of CD4 ${ }^{+}$IELs in day 8-infected mice in 3 independent experiments ( $\left.{ }^{\star} P<0.05\right)$. (d) TCR $\alpha \beta$ and TCR $\gamma \delta$ expression on CD4 ${ }^{+}$and CD $8 \alpha^{+}$IELs, respectively, from day 8-infected WT mice. IEL, intraepithelial lymphocyte; TCR, T-cell receptor; WT, wild type.

\section{Both IEL and LP compartments are required for maximal intestinal inflammation}

An important consideration for the adoptive transfer experiments in $\mathrm{Crr} 2^{-/-}$mice was the potential contribution of host LP cells to the development of intestinal lesions. Another consideration was the potential contribution of endogenous IEL in $\mathrm{Ccr} 2^{-/-}$recipients. A final complicating factor was that $\mathrm{C} c 2^{-/-}$ recipients were themselves infected with Toxoplasma. To avoid these complications, we tested the effects of transferring IELs and LP from day 4-infected WT mice into uninfected Rag1 ${ }^{-/-}$ mice that lack lymphocyte populations. We chose day 4 as the time point to isolate cells reasoning that at this time infection would be well established, but intestinal lesions would still be absent. As shown in Figure 2 and in ref. 13, IELs from day 4 -infected mice were composed of CD8 T cells expressing TCR $\alpha \beta$ and TCR $\gamma \delta$. The LP compartment from day 4-infected mice was a heterogeneous mix of $\mathrm{B}$ cells, $\mathrm{CD} 4^{+}$, and $\mathrm{CD} 8^{+} \mathrm{T}$ cells, dendritic cells, monocytes/macrophages, and a small number of neutrophils (Supplementary Figure S2A online). In accordance with other studies in which T. gondii induced a collapse in the regulatory T-cell population in the intestinal mucosa, ${ }^{19}$ we found no detectable Foxp3-positive cells in the IEL or LP compartment at this time point (data not shown). Similarly, our preparations contained very low numbers of NK and NKT cells (data not shown).

Both IEL and LP compartments were isolated from day 4 -infected WT mice, injected alone or in combination into
Rag1-/- recipients, and the small intestine collected 5 days later for histological evaluation. Noninfected Rag1 ${ }^{-/-}$mice displayed little or no evidence of intestinal lesions (Figure $4 \mathbf{a}$ and $\mathbf{b}$ ). Rag1 ${ }^{-/-}$recipients of the LP compartment also displayed minimal evidence of intestinal damage. Interestingly (given our previous transfer experiments in $\mathrm{Ccr}^{-/-}$mice), adoptive transfer of the IEL compartment alone induced only minor inflammation in $R a g 1^{-/-}$recipients, characterized mostly by epithelial tip sloughing. However, transfer of IELs in combination with LP triggered ileal lesions in $\mathrm{Rag1}^{-1-}$ recipients (Figure 4a and $\mathbf{b}$ ), and the pathology closely resembled that observed in infected WT mice (Supplementary Figure S1 online).

Increased adherence and translocation of luminal bacteria into the mucosal tissue are features of small intestinal lesions in Toxoplasma-infected mice. ${ }^{9}{ }^{10}$ Accordingly, we used fluorescence in situ hybridization using a probe specific for bacterial 16 S ribosomal RNA to assess translocation of the gut flora after adoptive transfer in Rag1 $1^{-/-}$animals. We found no evidence of bacterial translocation after transfer of IELs or LP into Rag1 ${ }^{-/-}$recipients (Figure 4c). In marked contrast, co-transfer of IEL and LP cells resulted in increased bacterial translocation into the LP regions of the $R a g 1^{-/-}$small intestine (Figure 4c).

We considered the possibility that lesions in $R a g 1^{-/-}$recipients were caused by co-transfer of parasites rather than IEL and LP cells per se. Accordingly, infection levels were assessed in the transfer populations after cell permeabilization and antibody 

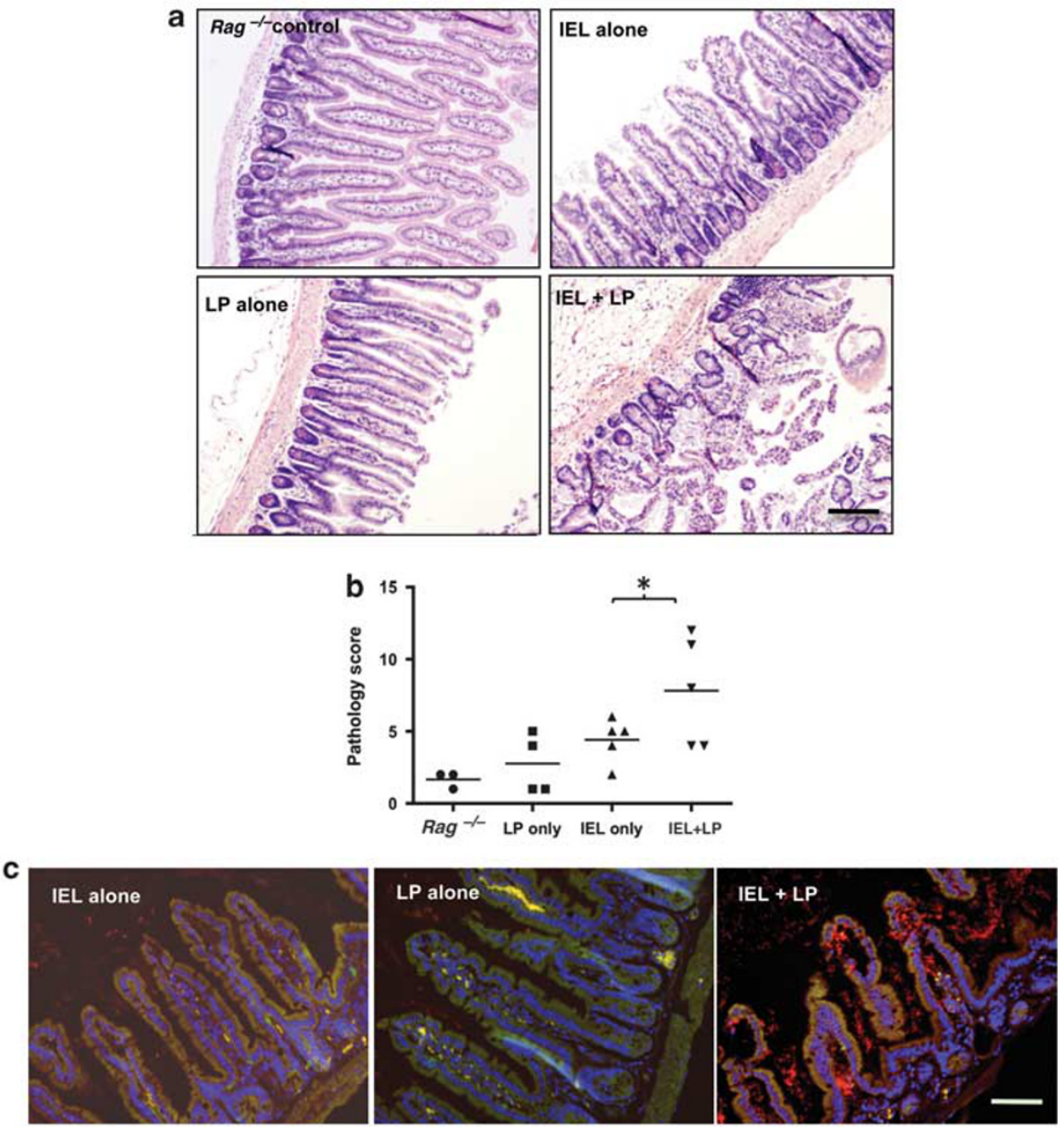

Figure 4 IELs and LP from infected mice synergize to induce intestinal lesions and bacterial translocation after transfer into uninfected Rag ${ }^{-/-}$ recipients. IELs and LP were isolated from day 4-infected WT mice and transferred alone or in combination into Rag $1^{-/-}$mice. Intestines were harvested 5 days after transfer, fixed, stained, and examined for inflammatory changes. Groups of noninfected Rag $1^{-/-}$mice received no WT cells, LP alone, IEL alone, or equal numbers of the two cell types (a). Scale bar=50 $\mu \mathrm{m}$. Pathology scores from individual mice in each group are shown in (b), ${ }^{\star} P<0.05$. The experiment was repeated twice with the same result. Noninfected $R a g-1^{-1-}$ animals received IELs, LP, or IEL +LP from day 4 -infected WT mice. Small intestines were collected 5 days after transfer and tissue sections were subjected to fluorescence in situ hybridization (FISH) analysis using a Cy3-labeled $16 \mathrm{~S}$ ribosomal RNA pan-bacterial probe (red) to detect the presence and localization of intestinal bacteria (c). The staining also includes a 6FAM-labeled nonsense probe, so that nonspecific binding is visible as yellow fluorescence. Cell nuclei were counterstained with DAPI (blue). Scale bar=50 $\mu \mathrm{m}$. This experiment was performed three times with the same result. IEL, intraepithelial lymphocyte; LP, lamina propria; TCR, T-cell receptor; WT, wild type.

staining for intracellular T. gondii. Neither IELs (Supplementary Figure S2B online) nor LP (Supplementary Figure S2C online) contained detectable parasites. In contrast, the same Toxoplasmareactive antibody was highly effective in detecting infected cells in the peritoneal cavity of WT mice after intraperitoneal injection of Toxoplasma (Supplementary Figure S2D online). Furthermore, tissues prepared from $\mathrm{Rag1}^{-/-}$recipients of WT IELs and LP 5 days after adoptive transfer showed no evidence of infection by immunohistochemical staining for Toxoplasma, despite induction of lesions in the small intestine (Supplementary Figure S2E online and Figure 4a). This contrasted with anti-Toxoplasma staining of the small intestine of day 8 -infected WT mice, in which parasites were easily detected (Supplementary Figure S2F online). We also inoculated day 4 -infected LP and IEL samples onto human fibroblast monolayers and found no evidence of plaque formation even after 2 weeks of culture (data not shown). Importantly, Rag1 ${ }^{-/-}$mice did not develop intestinal lesions after direct T. gondii infection (Supplementary Figure S3B online, compare with WT animals infected with the same parasite preparation in Supplementary Figure S3A online), despite the presence of parasites in the mucosa (Supplementary Figure S3C online). We conclude that intestinal lesions in Rag1 ${ }^{-/-}$ recipients of IEL and LP cells were not generated as a result of co-transfer of parasites, but instead as a result of synergistic interactions between these two cell populations.

\section{Rag $^{-/-}$IEL/LP recipients possess ileal cytokine profiles closely matching infected WT mice}

To examine the cytokine profile in $R a g 1^{-/-}$animals receiving IEL + LP, we cultured ileal biopsies and measured cytokine levels 

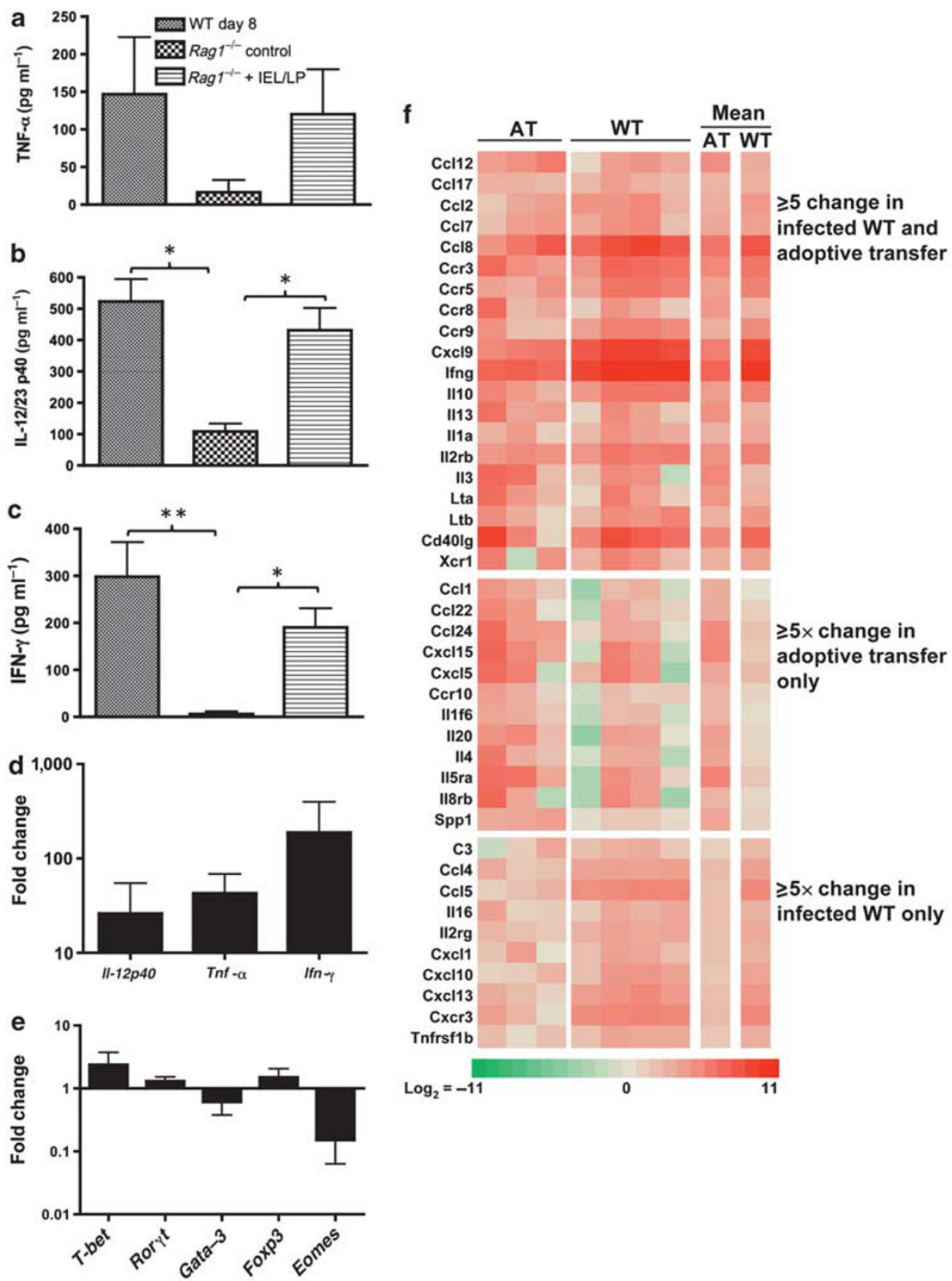

Figure 5 Reconstitution of Rag1//- mice with IELs and LP from infected WT mice induces a proinflammatory cytokine profile in recipient animals. Ileal explants from day 8-infected WT (excluding Peyer's patches), naive Rag1-/-, and Rag $1^{-/-}$IEL/LP recipients were cultured overnight and levels of (a) TNF- $\alpha$, (b) IL-12/23p40, and (c) IFN- $\gamma$ were determined by ELISA. For Rag $1^{-/-}$adoptively transferred recipients, IELs and LP were prepared from day 4-infected WT mice, transferred into noninfected Rag $1^{-/-}$mice, and ileal biopsy samples were isolated 5 days post transfer. RNA was isolated from ileal tissues of adoptively transferred mice and examined by real-time RT-PCR for II-12p40, Tnf- $\alpha$, and Ifn- $\gamma$ (d), as well as T-bet, Ror 7 , Gata-3, Foxp3, and Eomes (e). The data are plotted as change relative to samples from normal Rag $1^{-1-}$ mice. These experiments were repeated twice with similar results. lleal samples were also analyzed for proinflammatory gene transcripts in a real-time RT-PCR gene array (f). Transcripts displaying an average of fivefold or greater over uninfected Rag $1^{-/-}$ileal samples are represented as a clustering image. AT, Rag $1^{-/-}$recipients of LP/IEL; WT, day 8-infected WT ilea. Each column represents an individual mouse, with the last two columns representing mean values of the AT and WT groups relative to noninfected Rag1-/- ileal samples. ELISA, enzyme-linked immunosorbent assay; IEL, intraepithelial lymphocyte; IFN- $\gamma$, interferon- $\gamma$; IL, interleukin; LP, lamina propria; RT-PCR, reverse transcriptase-PCR; TCR, T-cell receptor; TNF- $\alpha$, tumor necrosis factor- $\alpha$; WT, wild type, ${ }^{\star} P>0.05 ;{ }^{* *} P>0.01$.

in the resulting supernatants. Ileal samples from day 8 -infected WT mice released substantial amounts of IFN- $\gamma$, IL-12/23p40, and TNF- $\alpha$ (Figure 5a-c). Ileal samples obtained from naive Rag1 ${ }^{-/-}$mice released low levels of each respective cytokine. In marked contrast, ilea from Rag1-/- IEL/LP recipients secreted IFN- $\gamma$, IL-12/23p40, and TNF- $\gamma$ at levels similar to tho se measured in WT samples (Figure 5a-c). We also examined transcriptional profiles and found that Il-12p40,Tnf- $\alpha$, and Ifn- $\gamma$ genes 
were each highly upregulated after IEL/LP transfer relative to ileal samples from nontransferred Rag1 ${ }^{-/-}$mice (Figure 5d). Analysis of the transcription factors in IEL/LP Rag-1 ${ }^{-1-}$ recipients revealed a pattern broadly similar to that seen in infected WT mice (Figures 1 and 5e).

We next used a real-time reverse transcriptase-PCR gene array targeted to inflammatory cytokines, chemokines, and their receptors to analyze transcriptional responses in the ilea of Rag1 $1^{-/-} / \mathrm{IEL}+\mathrm{LP}$ mice in comparison with infected WT mice and naive Rag1 ${ }^{-/}$control animals. The results are summarized as a heat map of the genes with greater than or equal to five-fold change over noninfected $\operatorname{Rag} 1^{-/-}$values (Figure 5f). Applying this threshold, 20 gene transcripts were upregulated in both the infected WT ileum and the ileum from AT Rag1 $1^{-/-}$recipients. In all, 12 transcripts were upregulated fivefold or more only in AT recipients, whereas 10 transcripts were upregulated to similar levels only in the infected WT ileum (Figure 5f). Among the common transcripts, Ifn- $\gamma$ was highly upregulated. In both WT and AT recipients, we also detected increased CCL2 and CCL8, major ligands for CCR2. The latter chemokine receptor is implicated in the development of ileal inflammation during Toxoplasma infection. ${ }^{13,17}$ Transcripts encoding several other chemokines and receptors involved in Th-1 inflammatory responses (CCL7, CCR3, CCR5, CCR9, CXCL9) were also elevated in both infected and Rag1-/- IEL/LP recipients.

Supplementary Figure S4 online shows linear scatter plots of the complete data sets. Relative to levels in control Rag1 $1^{-/-}$ mice, transcript levels in AT (Supplementary Figure S4A online) and infected WT mice (Supplementary Figure S4B online) remained within or were raised above the five-fold threshold, with no evidence for downregulation of transcripts. When transcript levels of AT vs. infected WT were compared, the majority of transcripts fell within the five-fold threshold, emphasizing the similarity of the ileal chemokine/cytokine environments in these two groups (Supplementary Figure S4C online). In summation, the chemokine/cytokine profile in $\mathrm{Rag1}^{-/-}$IEL/LP animals is remarkably similar to that of infected WT mice.

\section{Lesion development in Rag 1 $^{-/-}$recipients requires IELs but not LP from Toxoplasma-infected mice}

We next determined whether LP and IEL populations from noninfected WT mice were capable of inducing pathology after transfer into Rag1 ${ }^{-/-}$recipients. Adoptive transfer of IELs and LP from noninfected mice did not cause damage to the small intestine (Figure 6a). When IELs from noninfected mice were co-transferred with LP from infected animals, $R a g 1^{-/-}$recipients developed a low, but significant $(P<0.05)$ level of intestinal damage (Figure $\mathbf{6 b}$ and $\mathbf{d}$ ). However, by far, the most severe lesions were triggered by combined transfer of IELs from infected mice with LP from noninfected animals (Figure $\mathbf{6 c}$ and $\mathbf{d}$, $P<0.01)$. Pathology scores in the latter group were similar to scores obtained from transfers that were performed when both LP and IEL compartments were obtained from infected mice (Figure 4b).
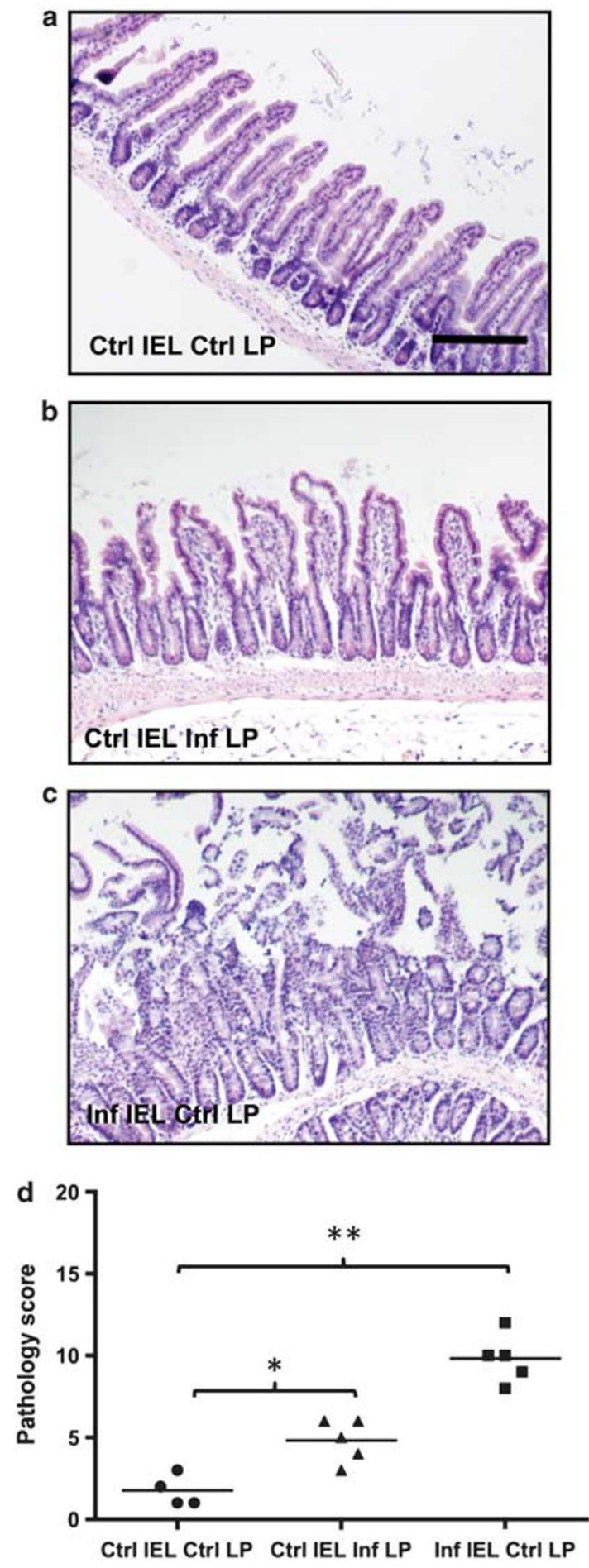

Figure 6 IELs from infected mice induce disease in Rag $1^{-/-}$mice when co-transferred with LP from noninfected animals. Noninfected Rag 1 $^{-/-}$recipients received combinations of IEL and LP from infected (Inf) and noninfected (Ctrl) mice, and then 5 days later tissues were collected for histopathological evaluation in the ileum. (a) Rag $1^{-/-}$mice receiving IELs and LP from noninfected mice; (b) Rag1-/- recipients of IELs from noninfected mice and LP from infected animals; (c) Rag $1^{-/-}$ recipients of IELs from infected mice in combination with LP from noninfected animals. (d) Scores for individual mice, ${ }^{*} P<0.05$ and ${ }^{\star \star} P<0.01$. Scale bar $=50 \mu \mathrm{m} ;$ (a). IEL, intraepithelial lymphocyte; LP, lamina propria. 
$\mathrm{CD}^{+} \mathrm{T}$ cells emerging in the IEL compartment derive from the LP and synergize with IELs to cause lesions

We used CD45 congenic animals to examine the dynamic behavior of LP cells after co-transfer with IELs into Rag1 ${ }^{-/-}$ mice. Groups of WT (CD45.2) and CD45.1 congenic mice were infected with $100 \mathrm{ME} 49$ cysts and the IEL and LP compartments were isolated 4 days later. Naive $R a g 1^{-/-}$recipients were injected with IELs from WT (CD45.2) mice along with LP from CD45.1 congenic animals. Five days after the adoptive transfer, IELs were harvested from $\mathrm{Ragl}^{-/-}$mice and stained with antibodies to determine the presence of $\mathrm{CD} 4^{+} \mathrm{T}$ cells and the LP congenic marker, CD45.1 as outlined in the schematic shown in Figure 7a. Transfer of LP alone resulted in little or no detectable CD $4{ }^{+}$cells in the IEL compartment (Figure 7b). However when IELs and LP were co-transferred, there was a 15 -fold increase in $\mathrm{CD} 4{ }^{+}$cells in the IEL compartment (with the remainder being epithelial cells; data not shown). Gating on $\mathrm{CD} 4{ }^{+}$cells revealed that the majority were CD45.1 positive and therefore derived from the LP compartment (Figure 7c). A smaller population of CD45.2-positive cells derived from transferred IELs was also present (Figure 7c). We conclude that IELs drive recruitment of $\mathrm{CD} 4{ }^{+}$cells in the LP, most likely through production of chemokines.

As $\mathrm{CD}^{+}{ }^{+} \mathrm{T}$ cells emerging in the IEL compartment express CCR2 (Figure 3b), we asked whether the presence of this receptor on $\mathrm{LPCD} 4^{+} \mathrm{T}$ lymphocytes was necessary to enable recruitment. CD45.2-positive cells could be recovered from the IEL compartment after transfer of WT CD45.2 LP along with WT CD45.1 IELs into Rag1 ${ }^{-/-}$recipients. We examined the phenotype of these cells and found that after co-transfer with WT LP cells, a population of CD45.2-positive CD4 ${ }^{+} \mathrm{T}$ cells appeared in the IEL compartment, and this population was significantly lower after co-transfer with $C c r 2^{-/-}$LP cells (Figure 7d). We conclude that CCR2 expression on LP CD4 ${ }^{+} \mathrm{T}$ cells is required for maximal recruitment to the IEL compartment.

Previous data have shown that $\mathrm{CD} 4{ }^{+} \mathrm{T}$ cells mediate intestinal pathology during Toxoplasma infection. Therefore, we asked whether $\mathrm{CD} 4{ }^{+} \mathrm{T}$ cells accounted for the pathogenic activity of the LP compartment when co-transferred with IELs. We isolated LP cells from day 4 -infected mice which in this experiment were composed of $\sim 23 \% \mathrm{CD}^{+}$and $15 \% \mathrm{CD} 8 \alpha^{+}$ T cells (Figure 8a). Using immunomagnetic beads, this population was separated into CD4-positive and CD4-negative fractions (Figure 8a). These populations were then each cotransferred along with day 4 -infected IELs and tissues were collected 5 days later. Although the overall ileal damage was relatively mild, we found that pathology tracked with the $\mathrm{CD} 4{ }^{+}$T-cell fraction (Figure $\mathbf{8 b}$ ) and not with the CD4-negative population of LP cells. We also observed an expansion in the intestinal flora in $\mathrm{CD}^{+}{ }^{+}$recipients, most of which was tightly associated with the mucosal surface (Figure 8c). This was in contrast to $\mathrm{CD} 4{ }^{-}$recipient mice. Here, we saw far less bacteria and there were minimal indications of bacterial tissue adherence (Figure 8c). In summation, these data show that CCR2-dependent $\alpha \beta \mathrm{TCR}^{+} \mathrm{CD} 8^{+}$IELs mediate recruitment of $\mathrm{LP}$-derived $\alpha \beta \mathrm{TCR}{ }^{+} \mathrm{CD} 4{ }^{+} \mathrm{T}$ cells into the epithelium resulting in damage to the intestinal tissue.

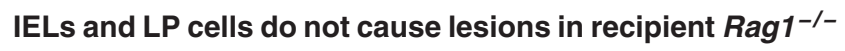 mice that have been treated with broad-spectrum antibiotics} Intestinal bacterial flora is recognized to have a role in the exacerbation of inflammation. Therefore, we asked whether the endogenous microbiota in recipient mice promoted the
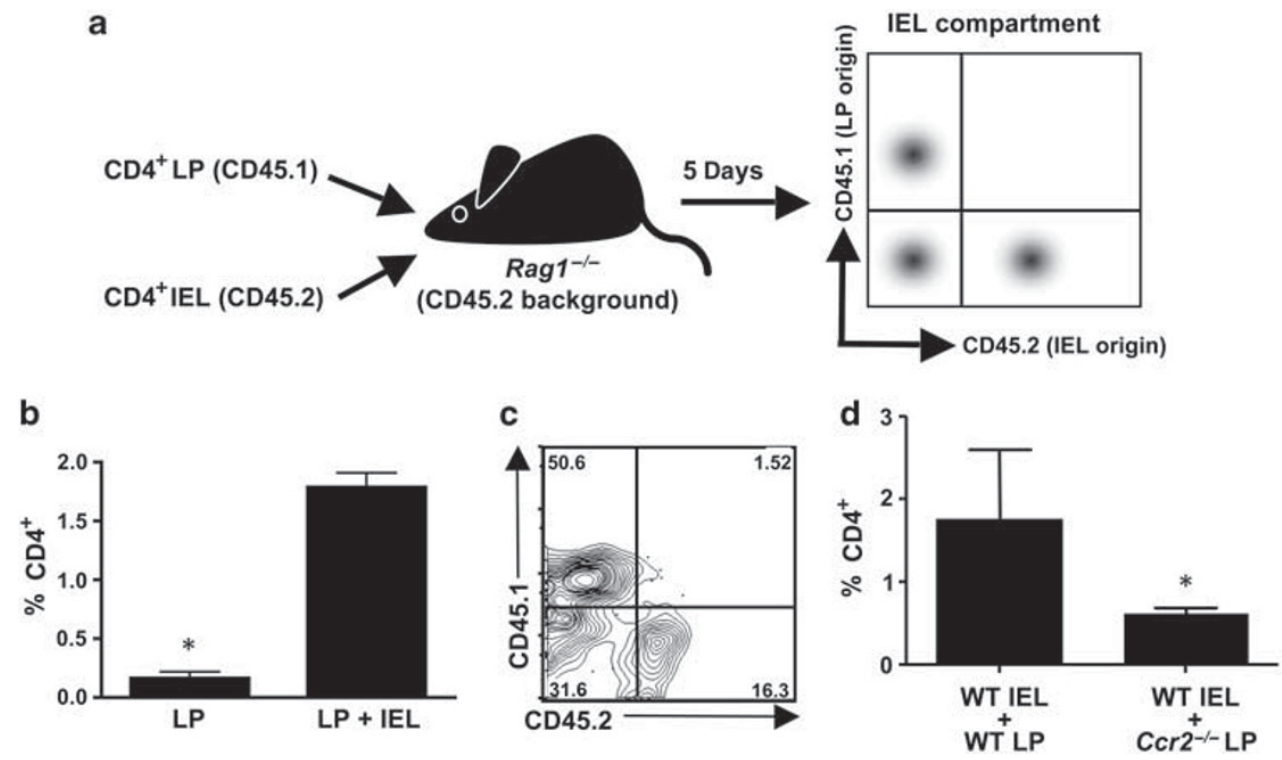

Figure 7 Parasite-elicited CD4+ LP lymphocytes are recruited into the IEL compartment in dependence on CCR2. (a) Uninfected Rag1-/- mice (CD45.2 background) were adoptively transferred with day 4-infected LP cells (Pep3\%/BoyJ; CD45.1) with or without day 4-infected IELs (C57BL/6; CD45.2) to trace the origin of cells emerging in the IEL compartment. (b) Five days after transfer, the IEL compartment was isolated and CD4 expression was examined in Rag1 ${ }^{-/-}$recipients of LP alone (LP) and LP in combination with IEL (LP+IEL). (c) Expression of CD45.1 (LP donor origin) and CD45.2 (IEL donor origin) by CD4 cells from mice receiving LP+IEL. (d) Percentage of CD4 ${ }^{+} \mathrm{T}$ cells in the IEL compartment in Rag1-/- recipients of WT IELs in combination with LP from WT or $C c r 2^{-/-}$mice. Donor cells were derived from day 4 -infected animals. ${ }^{*} P<0.05$. IEL, intraepithelial lymphocyte; LP, lamina propria; WT, wild type. 

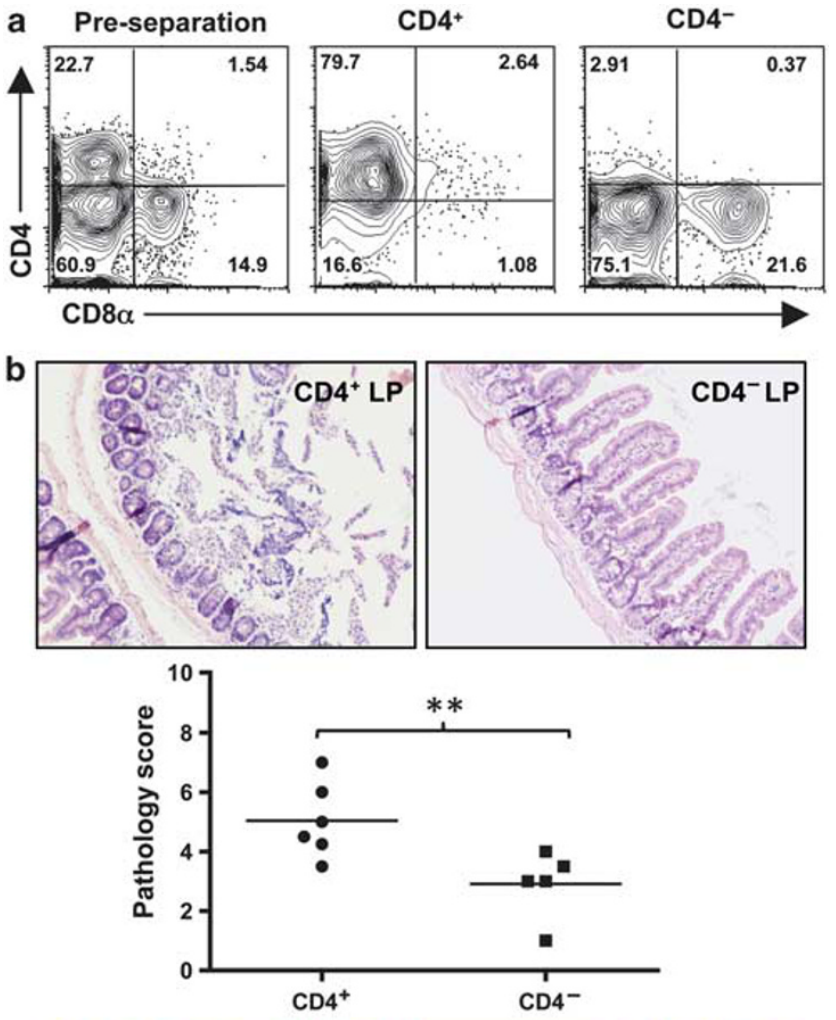

c

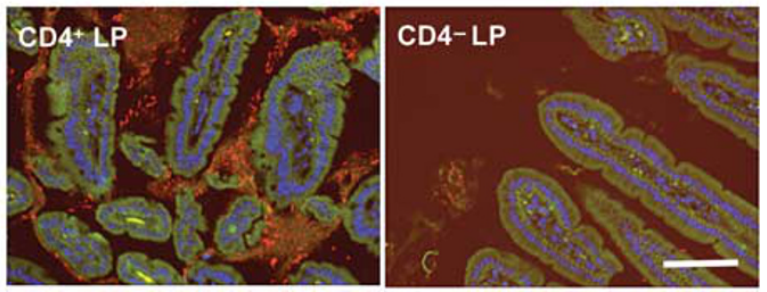

Figure 8 lleal lesion development and bacterial overgrowth are associated with CD4+ LP lymphocytes. Day 4-infected LP cells were fractionated using immunomagnetic beads into $\mathrm{CD} 4^{+}$and CD4- populations (a). Each was transferred with day 4-infected IELs into Rag 1-/- recipients and pathology assessed 5 days later. Representative ileal sections from a recipient of CD4 ${ }^{+} \mathrm{LP} / \mathrm{IEL}$ or a recipient of $C D 4^{-} \mathrm{LP} / \mathrm{IEL}$ and pathology scores of individual mice are shown in (b), ${ }^{* *} P<0.05$. FISH analysis using a $16 \mathrm{~S}$ ribosomal RNA pan-bacterial probe (red) was performed on ileal tissues from CD4 ${ }^{+} \mathrm{LP} / \mathrm{IEL}$ and CD4-LP/IEL Rag1-/- recipients (c). A 6FAM-labeled nonsense probe was included to control for nonspecific staining, and cell nuclei were stained with DAPI (blue). Scale bars $=50 \mu \mathrm{m}$. These experiments were repeated twice with similar results. FISH, fluorescence in situ hybridization; IEL, intraepithelial lymphocyte; LP, lamina propria.

pathogenic effector activity of IEL/LP transfer populations. Naive $\mathrm{Rag1}^{-/-}$mice were treated with a cocktail of broadspectrum antibiotics before and after adoptive transfer of IEL/LP. The effectiveness of the antibiotic treatment regimen was confirmed by Luria Bertani agar cultures of fecal pellets isolated at the termination of the experiment (Supplementary Figure S5 online).

Ileal damage was minimal in antibiotic-treated mice that were recipients of IEL/LP cells (Figure 9a and c) when compared with lesions in bacteria-replete mice receiving IEL/LP cells (Figure $\mathbf{9 b}$ and $\mathbf{c})$. Importantly, antibiotic treatment itself did not induce pathological changes in the intestines (Figure 9c). As a confirmation of disease induction in the intestine, biopsy cultures from antibiotic-treated mice receiving IEL/LP showed dramatically lower levels of IFN- $\gamma$ (Figure 9d) and TNF- $\alpha$ (Figure 9e) compared with nontreated animals receiving IEL/LP cells. We conclude that intestinal flora in recipient mice has a role in promoting the tissue-damaging effects of adoptively transferring IELs and LP cells.

\section{DISCUSSION}

Toxoplasma triggers proinflammatory lesions in the intestine of C57BL/6 mice. This destructive pathology has many features of Crohn's disease, insofar as both encompass ileal involvement, CD4 ${ }^{+}$T-lymphocyte pathogenic activity, inflammatory cytokine overproduction, and dysbiosis of gut microflora. ${ }^{12}$ Moreover, both Crohn's inflammation and Toxoplasma-triggered ileitis can be ameliorated by blocking TNF- $\alpha .{ }^{7,20}$ Recently, we uncovered a role for CCR2- and IFN- $\gamma$-dependent CD8 $\alpha^{+}$IELs in gut lesion development during oral T. gondii infection. ${ }^{13}$ Here, we establish that $\alpha \beta$ and not $\gamma \delta \mathrm{TCR}^{+}$IELs mediate intestinal damage. We also show that transfer of IELs together with LP cells from infected mice into naive Rag1 ${ }^{-/-}$mice triggers proinflammatory intestinal pathology in the absence of parasites. We show that IELs recruit LP-derived $\alpha \beta \mathrm{TCR}^{+} \mathrm{CD} 4^{+} \mathrm{T}$ cells into intraepithelial regions in partial dependence on CCR2, and that these cells are mediators of intestinal damage. Transfer of IELs alone in this model induced mild inflammation, suggesting that in addition to recruitment of LP cells, they may also directly contribute to damage in the intestine. We also found that endogenous intestinal flora in recipient mice was required for the pathogenic effects of cell transfer. These results, which are summarized in Supplementary Figure S6 online, are the first to demonstrate that microbial-induced interactions between IELs and LP lymphocytes promote proinflammatory cytokine responses and inflammatory lesions in the small intestine.

Important issues that await resolution are the major histocompatibility complex requirements and antigen specificity of the IEL and LPL subsets. The requirement for gut flora in recipient Rag1-/- mice suggests that one or both subsets could be reactive to intestinal bacteria. With regard to the IEL compartment, some CD8 ${ }^{+}$IEL subsets display unusual major histocompatibility complex requirements, ${ }^{16}$ and it will therefore be important in the future to examine responses of major histocompatibility complex class I and $\beta 2$-microglobulin knockout mice.

Our results show pathological activity of IELs induced by Toxoplasma. Nevertheless, under homeostatic conditions, the IEL compartment is generally associated with immunoregulation and immune quiescence in the intestinal mucosa. ${ }^{16,21}$ For example, transfer of CD8 $\alpha \alpha$ TCR $\alpha \beta$ IELs protects immunodeficient mice from subsequent transfer of pathogenic CD $4{ }^{+} \mathrm{CD} 45 \mathrm{RB}^{\text {high }}$ splenocytes. ${ }^{22}$ In parallel, it has been shown that $\gamma \delta \mathrm{TCR}^{+}$IELs have a protective function in the dextran sodium sulfate mouse colitis model. ${ }^{23}$ Yet, IELs isolated from Crohn's disease patients have been shown to display abnormally enhanced cytotoxic 

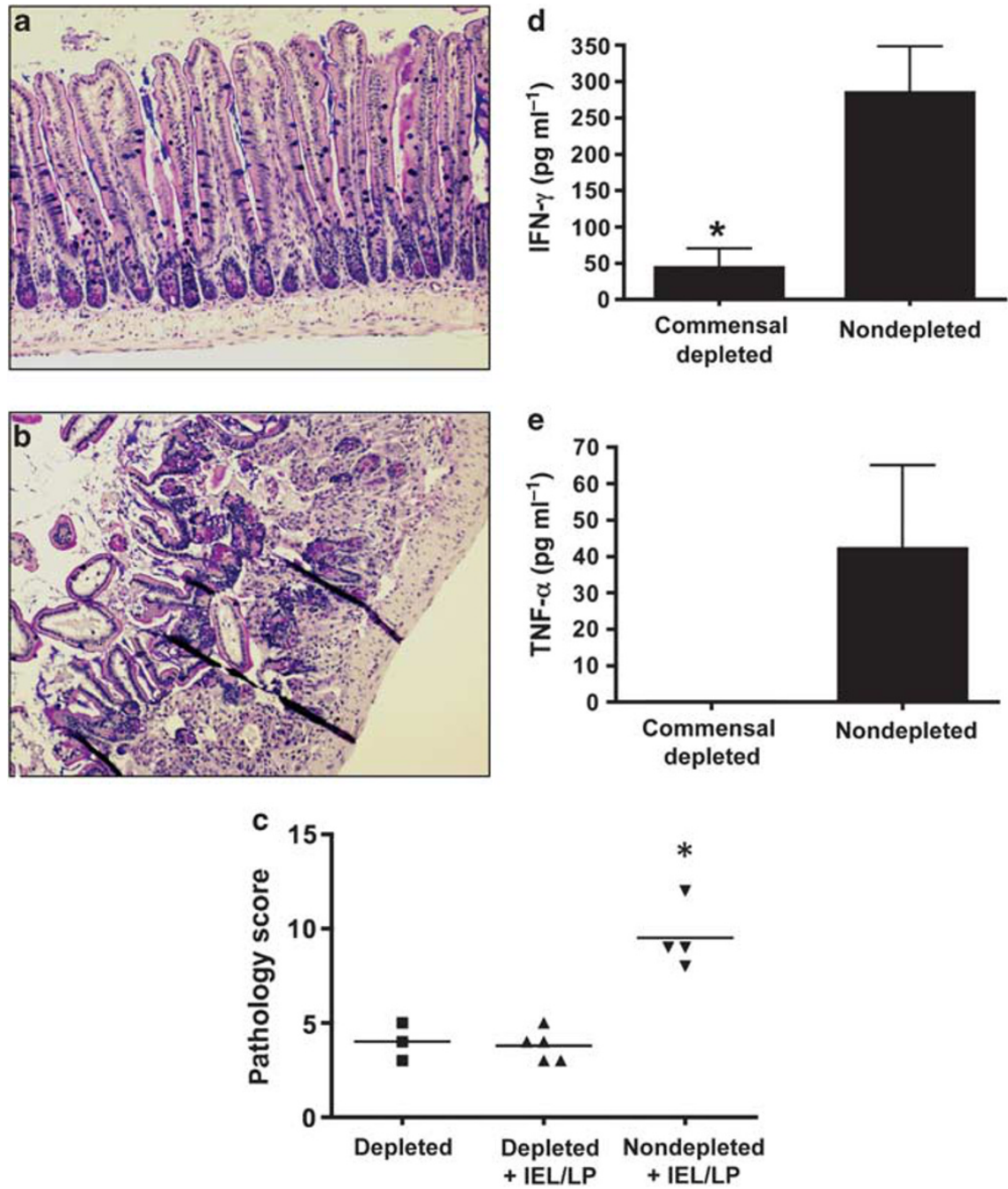

Figure 9 Elimination of intestinal flora in Rag1-/- mice prevents intestinal inflammation after IEL+LP transfer. Recipient mice were pre-treated with a broad-spectrum antibiotic cocktail for 3 weeks before cell transfer. Day 4 parasite-elicited IEL/LP populations were subsequently adoptively transferred into recipients and intestinal inflammation and cytokine production was evaluated 5 days later. (a) Rag $1^{-/-}$recipients treated with broadspectrum antibiotics and adoptively transferred with LP/IEL. (b) In parallel, nontreated Rag $1^{-/-}$animals received the same populations of IEL/LP. As depletion of flora results in mucous overproduction and interference with H\&E staining, tissue sections in panels $\mathbf{a}$ and $\mathbf{b}$ were stained with Alcian blue/PAS. (c) Pathology scores for commensal depleted, commensal depleted with IEL/LP cells, and noncommensal depleted with IEL/LP cells. Each symbol represents a single mouse. (d) IFN- $\gamma$ and (e) TNF- $\alpha$ production in intestinal biopsy samples obtained from commensal depleted or nondepleted $R$ Rag $1^{-/-}$recipients of IEL/LP populations. In these experiments, ${ }^{*} P<0.05$. These experiments were repeated twice with similar results. $\mathrm{H} \& \mathrm{E}$, hematoxylin and eosin; IEL, intraepithelial lymphocyte; IFN- $\gamma$, interferon- $\gamma$; LP, lamina propria.

activity and overproduction of IFN- $\gamma$ compared with IELs from normal donors. ${ }^{24,25}$ The latter observations, together with our data, suggest that dysfunctional IELs form part of the constellation that constitutes IBD.

Related to our studies is elegant work by others who examined early mucosal responses during low-dose Toxoplasma infection, a situation that avoids immunopathology, enabling host survival, protective immunity, and persistent infection. Here, IELs protect against ileitis and subsequent parasite challenge after adoptive transfer. ${ }^{26}$ In the LP compartment, $\mathrm{CD} 4{ }^{+} \mathrm{T}$ cells synergize with intestinal epithelial cells resulting in protective proinflammatory responses that control infection. ${ }^{27}$ At least part of the beneficial activity of IELs in this model stems from transforming growth factor- $\beta$ production that downregulates LP CD4 ${ }^{+} \mathrm{T}$-lymphocyte responses and controls ileitis. ${ }^{28,29}$ We hypothesize that highdose T. gondii infection triggers a switch in IEL function from mediators of protection and immunoregulation to effectors of pathology.

In our model, transfer of IEL and LP cells from Toxoplasmainfected mice triggered fulminant pathology in noninfected Rag1 ${ }^{-/-}$recipients. It seems most unlikely that parasites were the direct cause of disease in these mice as we could not detect parasites in either cells injected into the naive $\mathrm{Rag1}^{-/}$or in intestinal tissue isolated 5 days after transfer. Furthermore, $\mathrm{Rag1}^{-/-}$mice are resistant to lesion development when directly infected with T. gondii (Supplementary Figure S3 online and 
ref. 30). In WT mice, development of intestinal lesions during Toxoplasma infection is associated with a shift from Grampositive to predominantly Gram-negative bacteria in the small intestine, and fulminant pathology is accompanied by increased adherence and bacterial translocation into the subepithelium. Host responses to gut bacteria are implicated in intestinal inflammation because both $\mathrm{Tlr} 4^{-/-}$and $\mathrm{Tlr} \mathrm{9}^{-/-}$mice are resistant to lesion development. ${ }^{10,11,31}$

We show here that $C \mathrm{cr} 2^{-/-}$LP cells do not re-locate to the IEL compartment after transfer into Rag1 ${ }^{-/-}$mice. Yet, previously we found that IEL transfer into T. gondii-infected $\mathrm{Ccr}^{-/-}$ recipients (wherein the LP compartment would be CCR2 negative) was able to induce intestinal damage. We speculate that in the latter case, other chemokines and cytokines induced as a result of Toxoplasma infection in the recipients compensate for the loss of CCR2 responsiveness.

What is the triggering event that drives Toxoplasma-mediated intestinal lesion development? Possibly, the parasite induces early barrier damage as a result of invasion and egress from epithelial cells, enabling translocation of bacteria and in turn triggering pathogenic responses. Arguing against this scenario are data suggesting that Toxoplasma crosses the intestinal barrier using a paracellular pathway that involves transmigration without membrane disruption. ${ }^{32}$ It is also possible that the parasite directly activates mucosal lymphocytes for pathogenic activity, although this does not fully account for the requirement for bacterial flora in lesion development.

Another possibility is that disease development is a consequence of immunosuppression by Toxoplasma. The parasite is known to downregulate cytokine and chemokine responses during intracellular macrophage and dendritic cell infection. ${ }^{33-36}$ This may have relevance to induction of IBD, because recent data have revealed impaired cytokine secretion by macrophages from Crohn's patients. ${ }^{37}$ Older studies have shown impaired neutrophil recruitment in individuals with Crohn's disease. ${ }^{38,39}$ Thus, a model for Crohn's pathogenesis is that defective sentinel responses to occasional bacterial ingress in the gut results in failure to recruit neutrophils, leading to loss of control of infection and chronic secondary inflammation. ${ }^{37}$ We are currently examining the functional status of intestinal dendritic cells and macrophages at early time points of T. gondii infection.

The results of this study highlight the value of Toxoplasma infection in C57BL/6 mice as a model for induction of Crohn'slike pathology in the ileum. Our results reveal for the first time that LP T cells and IELs synergize to cause inflammatory ileitis. By exploiting Toxoplasma as a trigger, we can distinguish cell and cytokine requirements required for inflammation, and in so doing we can expect to gain further mechanistic insights into immunological factors that are important in human IBDs.

\section{METHODS}

Mice. Female C57BL/6 and Swiss Webster mice, 8-12-weeks old, were purchased from Taconic Farms (Germantown, NY). B6.SJL-

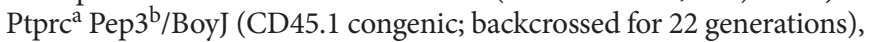

B6.129S7-Rag1 $1^{\mathrm{tm} 1 \mathrm{Mom} / \mathrm{J}}\left(\mathrm{Rag1}^{-/-}\right.$; backcrossed for 10 generations), and B6.129S4-Ccr2 ${ }^{\mathrm{tm} 1 \mathrm{lfc}} / \mathrm{J}$ (Ccr2 ${ }^{-1-}$; backcrossed for 9 generations) mice were purchased from The Jackson Laboratory (Bar Harbor, ME). Animals were housed under specific pathogen-free conditions at the Cornell University College of Veterinary Medicine animal facility, which is accredited by the International Association for Assessment and Accreditation of Laboratory Animal Care.

Antibiotic treatment. Mice were treated with sterile water supplemented with ampicillin $\left(\mathrm{g} \mathrm{l}^{-1}\right.$, Sandoz, Princeton, NJ), vancomycin (500 mg l-1, Hospira, Lake Forest, IL), enrofloxacin (200 $\mathrm{mg} \mathrm{l}^{-1}$, Bayer, Pittsburgh, PA), and imipenem (250 $\mathrm{mg} \mathrm{l}^{-1}$, Merck, Whitehouse Station, NJ) ad libitum for 3-4 weeks. Antibiotics were changed weekly and cages changed every 3 days.

Parasites and infections. Swiss-Webster mice were used to generate cysts of the low virulence type II Toxoplasma strain, ME49. Mice were infected with 100 cysts by oral gavage.

LP and IEL isolation. Small intestines were removed, cleaned of mesentery and fat, and flushed with sterile phosphate-buffered saline. Isolation of IELs was performed as described previously. ${ }^{13}$ Absence of contaminating LP cells was tested by antibody staining for B lymphocytes, which are absent from the IEL compartment. LP leukocytes were isolated as described previously. ${ }^{40}$ After removal of the mucosal layer, the small intestine was cut into $5 \mathrm{~mm}$ fragments. Cells were subsequently liberated from tissues by digestion $\left(37^{\circ} \mathrm{C}, 20 \mathrm{~min}\right)$ in RPMI containing $0.2 \mathrm{mg} \mathrm{ml}^{-1}$ Liberase CI (Roche, Nutley, NJ) and $15 \mathrm{mg} \mathrm{ml}^{-1}$ DNAse (Sigma-Aldrich, St Louis, MO). Leukocytes were separated from contaminating debris by discontinuous Percoll gradient centrifugation.

Lymphocyte isolation. For CD4 ${ }^{+} \mathrm{T}$-cell separation, single cell suspensions of LP leukocytes were incubated with anti-CD4 magnetic beads according to the manufacturer's instructions (Miltenyi Biotech, Auburn, CA). Cells were washed and separated by positive selection on an AutoMACs separator (Miltenyi Biotech). Similarly, $\alpha \beta$ IELs were separated from $\gamma \delta$ IELs using anti- $\gamma \delta$ magnetic beads (Miltenyi Biotech).

Gut biopsy culture. Intestines were removed from mice and flushed with sterile phosphate-buffered saline containing $300 \mathrm{U} \mathrm{ml}^{-1}$ penicillin and $300 \mu \mathrm{g} \mathrm{ml}^{-1}$ streptomycin. Intestines were opened longitudinally and a 6-mm dermal biopsy punch (Miltex, York, PA) was used to collect tissue pieces. Intestinal sections were incubated overnight in Dulbecco's modified Eagle's medium supplemented with $10 \%$ bovine growth serum, $1 \mathrm{~mm}$ sodium pyruvate, $0.1 \mathrm{~mm}$ nonessential amino acids, $0.05 \mathrm{~mm} \beta$-mercaptoethanol, $300 \mathrm{U} \mathrm{ml}^{-1}$ penicillin, $300 \mu \mathrm{g} \mathrm{ml}^{-1}$ streptomycin, and $30 \mathrm{~mm} \operatorname{HEPES}\left(37^{\circ} \mathrm{C}, 4 \% \mathrm{CO}_{2}\right)$. Cell-free supernatants were collected and cytokine levels measured as described previously. ${ }^{41}$

Flow cytometry. Single cell suspensions were stained in buffer (phosphate-buffered saline, $1 \%$ bovine serum albumin, $0.01 \% \mathrm{NaN}_{3}$ ) containing 10\% mouse serum. Anti-CCR2 (clone MC-21) was used as described previously. ${ }^{13}$ Antibodies used were phycoerythrin- and allophycocyanin-conjugated anti-CD4 mAb, fluorescein isothiocyanate- and allophycocyanin-conjugated anti-CD $\alpha \alpha \mathrm{mAb}$, phycoerythrin-conjugated anti-CD $8 \beta \mathrm{mAb}$, PerCP-Cy5.5-conjugated anti-TCR $\beta$, and fluorescein isothiocyanate- and allophycocyanin-conjugated anti-TCR $\gamma \delta$ (all from eBioscience, San Diego, CA). Anti-SAG-1 (p30) directly conjugated to fluorescein isothiocyanate (Argene, Shirley, NY) was used to stain intracellular parasites after permeabilization with $0.075 \%$ saponin. Cells were analyzed on a BD FACSCalibur flow cytometer (Franklin Lakes, NJ).

Adoptive transfer. Mice received $5 \times 10^{6}$ IELs, $5 \times 10^{6} \mathrm{LP}$, or a 1:1 mixture of both (totaling $1 \times 10^{7}$ cells) by intravenous retro-orbital injection. Mice 
receiving IELs and $\mathrm{CD} 4^{+}$or $\mathrm{CD} 4^{-} \mathrm{LP}$ received $5 \times 10^{6} \mathrm{IELs}$ and $1 \times 10^{6} \mathrm{LP}$. Intestines were subsequently removed and fixed in $10 \%$ neutral-buffered formalin. Groups of paraffin-embedded tissue sections were stained with hematoxylin and eosin or Alcian blue/periodic acid Schiff and examined for pathological changes, and sequential sections were subjected to fluorescence in situ hybridization.

RNA isolation, microarray analysis, and semi-quantitative PCR. RNA was prepared from 1-cm ileum segments using an RNA purification kit (Omega Bio-Tek, Norcross, GA). RNA was converted into cDNA following the manufacturer's recommendations (SABiosciences, Frederick, $\mathrm{MD}$ ), and samples were run on the mouse "Inflammatory Cytokines \& Receptors PCR Array" (SABiosciences) using an ABI 7500 Fast machine. Data were normalized to housekeeping genes present of the array and compared with uninfected Rag-1 knockout mice. Semi-quantitative PCR was performed using the ABI-Prism 7500 fast cycle real-time PCR system (Applied Biosystems, Carlsbad, CA). Gene expression levels were normalized to Gapdh expression and differences between groups were analyzed by the $\Delta \Delta C_{\mathrm{T}}$ method. Transcriptional responses were expressed relative to noninfected mice.

Fluorescence in situ hybridization. Fluorescence in situ hybridization was performed as described previously, ${ }^{13}$ using probes (EUB338 or nonEUB338) labeled on the $5^{\prime}$ end with either fluorescein isothiocyanate or Cy3 (Integrated DNA Technologies, Coralville, IA). Images were collected using a BX51 microscope (Olympus, Center Valley, PA) equipped with a DP70 camera using DP Controller Software (version 1.1.1.65; Olympus) and DP Manager software (version 1.1.1.71; Olympus).

Pathology scoring. Damage to the intestine was scored as described previously. ${ }^{13}$ In brief, five criteria (villus fusion/blunting, LP inflammation, epithelial tip sloughing, villus tip necrosis, and transmural inflammation) were each assigned a score of 0 (not apparent) to 4 (severe). Each mouse was assigned a cumulative score out of a maximum of 20 . Scoring was carried out in a double-blinded manner.

Statistical analyses. Pathology scores were analyzed using an ANOVA (analysis of variance) with a Bonferroni post test correction to calculate statistical differences between groups. Where appropriate, Student's $t$-test was used to analyze statistical differences between two groups. Values of $P<0.05$ were considered significant.

SUPPLEMENTARY MATERIAL is linked to the online version of the paper at http://www.nature.com/mi

\section{ACKNOWLEDGMENTS}

This work was funded by NIH grants to EYD (AI083526) and KJM (DK077728), and a CCFA Senior Fellowship Award (KWS). SBC was supported by the Cornell University Graduate Program in Biological and Biomedical Sciences. The technical assistance of F. Davis and M. Hossain is gratefully acknowledged.

\section{DISCLOSURE}

The authors declared no conflict of interest.

C 2011 Society for Mucosal Immunology

\section{REFERENCES}

1. Xavier, R.J. \& Podolsky, D.K. Unravelling the pathogenesis of inflammatory bowel disease. Nature 448, 427-434 (2007).

2. Baumgart, D.C. \& Carding, S.R. Inflammatory bowel disease: cause and immunobiology. Lancet 369, 1627-1640 (2007).

3. Kaser, A., Zeissig, S. \& Blumberg, R.S. Inflammatory bowel disease. Annu. Rev. Immunol 28, 573-621 (2010).

4. Dubey, J.P. The history and life-cycle of Toxoplasma gondii. In Toxoplasma gondii The Model Apicomplexan: Perspective and Methods. (Weiss, L.M. \& Kim, K., eds) 1-17 (Academic Press, San Diego, 2007).
5. Montoya, J.G. \& Liesenfeld, O. Toxoplasmosis. Lancet 363, 1965-1976 (2004).

6. Denkers, E.Y. \& Gazzinelli, R.T. Regulation and function of T cell-mediated immunity during Toxoplasma gondii infection. Clin. Microbiol. Rev. 11, 569-588 (1998).

7. Liesenfeld, O. et al. TNF-a, nitric oxide and IFN-g are all critical for development of necrosis in the small intestine and early mortality in genetically susceptible mice infected perorally with Toxoplasma gondii. Parasite Immunol. 21, 365-376 (1999).

8. Liesenfeld, O., Kosek, J., Remington, J.S. \& Suzuki, Y. Association of $\mathrm{CD}^{+} \mathrm{T}$ cell-dependent, IFN-g-mediated necrosis of the small intestine with genetic susceptibility of mice to peroral infection with Toxoplasma gondii. J. Exp. Med. 184, 597-607 (1996).

9. Heimesaat, M.M. et al. Gram-negative bacteria aggravate murine small intestinal Th1-type immunopathology following oral infection with toxoplasma gondii. J. Immunol. 177, 8785-8795 (2006).

10. Heimesaat, M.M. et al. Exacerbation of murine ileitis by toll-like receptor 4 meditated sensing of lipopolysaccharide from commensal Escherichia coli. Gut 56, 941-948 (2007).

11. Minns, L.A. et al. TLR9 is required for the gut-associated lymphoid tissue response following oral infection of toxoplasma gondii. J. Immunol. 176, 7589-7597 (2006)

12. Liesenfeld, O. Oral infection of C57BL/6 mice with Toxoplasma gondii: a new model of inflammatory bowel disease? J. Infect. Dis. 185, S96-S101 (2002).

13. Egan, C.E. et al. CCR2-dependent intraepithelial lymphocytes mediate inflammatory gut pathology during Toxoplasma gondii infection. Mucosal. Immunol. 2, 527-535 (2009).

14. El-Asady, R. et al. TGF-\{beta\}-dependent CD103 expression by CD8(+) $T$ cells promotes selective destruction of the host intestinal epithelium during graft-versus-host disease. J. Exp. Med. 201, 1647-1657 (2005).

15. Hue, S. et al. A direct role for NKG2D/MICA interaction in villous atrophy during celiac disease. Immunity 21, 367-377 (2004).

16. Kunisawa, J., Takahashi, I. \& Kiyono, H. Intraepithelial lymphocytes: their shared and divergent immunological behaviors in the small and large intestine. Immunol. Rev. 215, 136-153 (2007).

17. Benevides, L. et al. CCR2 receptor is essential to activate microbicidal mechanisms to control Toxoplasma gondii infection in the central nervous system. Am. J. Pathol. 173, 741-751 (2008).

18. Uhlig, H.H. \& Powrie, F. The role of mucosal T lymphocytes in regulating intestinal inflammation. Springer Semin. Immunopathol. 27, 167-180 (2005).

19. Oldenhove, G. et al. Decrease of Foxp3+ Treg cell number and acquisition of effector cell phenotype during lethal infection. Immunity 31, 772-786 (2009).

20. Ferrante, M. et al. Efficacy of infliximab in refractory pouchitis and Crohn's disease-related complications of the pouch: a Belgian case series. Inflamm. Bowel. Dis. 16, 243-249 (2010).

21. Ishikawa, H. et al. Curriculum vitae of intestinal intraepithelial $T$ cells: their developmental and behavioral characteristics. Immunol. Rev. 215, 154-165 (2007).

22. Das, G. et al. An important regulatory role for $\mathrm{CD} 4+\mathrm{CD} 8$ alpha alpha $\mathrm{T}$ cells in the intestinal epithelial layer in the prevention of inflammatory bowel disease. Proc. Natl Acad. Sci. USA 100, 5324-5329 (2003).

23. Chen, Y., Chou, K., Fuchs, E., Havran, W.L. \& Boismenu, R. Protection of the intestinal mucosa by intraepithelial gamma delta T cells. Proc. Natl Acad. Sci. USA 99, 14338-14343 (2002).

24. Nussler, N.C. et al. Enhanced cytolytic activity of intestinal intraepithelial lymphocytes in patients with Crohn's disease. Langenbecks Arch. Surg. 385, 218-224 (2000).

25. Watanabe, M. et al. Preferential activation of CD4+V beta 5.2/5.3+ intestinal intraepithelial lymphocytes in the inflamed lesions of Crohn's disease. Clin. Immunol. Immunopathol. 78, 130-139 (1996).

26. Lepage, A.C., Buzoni-Gatel, D., Bout, D.T. \& Kasper, L.H. Gut-derived intraepithelial lymphocytes induce long term immunity against Toxoplasma gondii. J. Immunol. 161, 4902-4908 (1998).

27. Mennechet, F.J.D. et al. Lamina propria CD4+ T lymphocytes synergize with murine intestinal epithelial cells to enhance proinflammatory response against an intracellular pathogen. J. Immunol. 168, 2988-2996 (2002). 


\section{ARTICLES}

28. Buzoni-Gatel, D. et al. Murine ileitis after intracellular parasite infection is controlled by TGF-b-producing intraepithelial lymphocytes. Gastroenterology 120, 914-924 (2001).

29. Mennechet, F.J. et al. Intestinal intraepithelial lymphocytes prevent pathogen-driven inflammation and regulate the Smad/T-bet pathway of lamina propria CD4+ T cells. Eur. J. Immunol. 34, 1059-1067 (2004).

30. Rachinel, N. et al. The induction of acute ileitis by a single microbial antigen of Toxoplasma gondii. J. Immunol. 173, 2725-2735 (2004).

31. Benson, A., Pifer, R., Behrendt, C.L., Hooper, L.V. \& Yarovinsky, F. Gut commensal bacteria direct a protective immune response against Toxoplasma gondii. Cell Host Microbe 6, 187-196 (2009).

32. Barragan, A. \& Sibley, L.D. Transepithelial migration of Toxoplasma gondi is linked to parasite migration and virulence. J. Exp. Med. 195, 1625-1633 (2002).

33. Bierly, A.L., Shufesky, W.J., Sukhumavasi, W., Morelli, A. \& Denkers, E.Y. Dendritic cells expressing plasmacytoid marker PDCA-1 are Trojan horses during Toxoplasma gondii infection. J. Immunol. 181, 8445-8491 (2008).

34. Lee, C.W., Bennouna, S. \& Denkers, E.Y. Screening for Toxoplasma gondii regulated transcriptional responses in LPS-activated macrophages. Infect. Immun. 74, 1916-1923 (2006).
35. Luder, C.G.K., Algner, M., Lang, C., Bleicher, N. \& Gross, U. Reduced expression of the inducible nitric oxide synthase after infection with Toxoplasma gondii facilitates parasite replication in activated murine macrophages. Int. J. Parasitol. 33, 833-844 (2003).

36. McKee, A.S., Dzierszinski, F., Boes, M., Roos, D.S. \& Pearce, E.J. Functional inactivation of immature dendritic cells by the intracellular parasite Toxoplasma gondii. J. Immunol. 173, 2632-2640 (2004).

37. Smith, A.M. et al. Disordered macrophage cytokine secretion underlies impaired acute inflammation and bacterial clearance in Crohn's disease. J. Exp. Med. 206, 1883-1897 (2009).

38. Marks, D.J. et al. Defective acute inflammation in Crohn's disease: a clinical investigation. Lancet 367, 668-678 (2006).

39. Segal, A.W. \& Loewi, G. Neutrophil dysfunction in Crohn's disease. Lancet 2, 219-221 (1976).

40. Lefrancois, L. \& Lycke, N. Isolation of mouse small intestinal intraepithelial lymphocytes, Peyer's patch, and lamina propria cells. Curr. Protoc. Immunol. Chapter 3, Unit 3 19, 2-16 (2001).

41. Sukhumavasi, W. et al. TLR adaptor MyD88 is essential for pathogen control during oral toxoplasma gondii infection but not adaptive immunity induced by a vaccine strain of the parasite. J. Immunol. 181, 3464-3473 (2008). 